\title{
Rhabdomyolysis and Obstetric Correlates: Literature Perspectives for the Obstetric Anesthesiologist
}

\author{
Rabiu Momoh
}

\section{ABSTRACT}

\begin{abstract}
Rhabdomyolysis complicating the antepartum or peripartum period is not a commonly reported finding and where they occur, it can be life-threatening. The problem with rhabdomyolysis during pregnancy or labour is the potential harmful systemic and local effects it can cause. These includes cardiac arrhythmias, acute kidney injury, clotting problems in severe cases [1]. To the fetus, foetal distress or foetal demise could be the end-result where timely management is not initiated [2].
\end{abstract}

Keywords: Creatine kinase, gluteal compartment syndrome, labour, neuraxial analgesia, pregnancy, rhabdomyolysis.

\section{INTRODUCTION}

Common causes of rhabdomyolysis known include severe exercises, crush injuries, burns, falls, prolonged immobilization, tetanus, status epilepticus, drug-induced (statins), malignant hyperthermia among other causes. Certain infections (e.g., Coxsackie virus, Epstein Barr virus, Legionella pneumophila, Salmonella, Plasmodium falciparum), disorders of muscle metabolism and certain auto-immune states involving muscles have also been identified as a predisposing factor for the development of rhabdomyolysis in patients [3]. The causes listed afore could potentially find relation as an obstetric emergency or comorbidity complicating the antepartum or peripartum period and the obstetric anesthesiologist should be aware.

Reference [4] in their presentation at the 2019 New York State Society of Anesthesiologists' Postgraduate Assembly described the case of a 32-year-old female who developed gluteal and hip muscle groups pain after the withdrawal of labour epidural analgesia for a spontaneous vaginal twin delivery (with evident raised serum creatinine kinase and lactate dehydrogenase levels that lowered with intravenous hydration). Prolonged immobility was identified as a predisposing factor for her rhabdomyolysis. There were no severe complications like acute kidney injury, cardiac arrhythmias, compartment syndrome or hematologic conditions like disseminated intravascular coagulopathy associated with the case they reported [4].

Acute kidney injury associated with rhabdomyolysis could be due to myoglobin inducing renal vasoconstriction, causing intratubular casts or through its direct toxicity to tubular cells in the kidneys (acute tubular necrosis) [5]. The cardiac arrhythmias that can complicate severe rhabdomyolysis are thought to be due to those of severe hyperkalemia or severe hypocalcemia that is known with severe rhabdomyolysis [6].
Submitted : October 6, 2021

Published : November 8, 2021

ISSN: $2593-8339$

DOI: $10.24018 /$ ejmed.2021.3.6.1098

\section{R. Momoh*}

Department of Intensive Care Medicine, William Harvey Hospital, United Kingdom.

(e-mail: rabiu.momoh@nhs.net)

*Corresponding Author

Reference [7] reported a case of a woman at 37 weeks gestational age, who developed muscle weakness, had severe hypokalemia $(2.0 \mathrm{mmol} / \mathrm{l})$, was noted with increased urinary potassium loss, had a trough serum bicarbonate value of $16 \mathrm{mmol} / 1$ and a peak creatinine kinase value of 5,338 IU/L. An assessment of rhabdomyolysis due to severe hypokalemia from distal renal tubular acidosis was made and patient was managed with fluid therapy and had potassium and bicarbonate replacement.

Reference [8] published a case series of rhabdomyolysis in four pregnant patients which included the case reported by Srisuttayasathien M. The unifying diagnosis in the four case reports in their series was rhabdomyolysis due to severe hypokalemia from distal renal tubular acidosis.

Medications (such as statins, fibrates, certain antipsychotics, selective serotonin reuptake inhibitors (SSRIs), diuretics) have been associated with their tendency to cause rhabdomyolysis including succinylcholine and inhalational anaesthetics [9]. Reference [10] reported a case of rhabdomyolysis with onset of symptoms an hour after epidural steroid (dexamethasone) administration to treat a chronic low back pain in a 47-year-old female. Serum creatinine kinase value up to $20,123 \mathrm{U} / \mathrm{L}$ (26-140), serum myoglobin $2977 \mathrm{ng} / \mathrm{ml}$ (14-66). There was resolution of her symptoms and biomarkers after ten days with intravenous fluid therapy in an intensive care unit [10].

Reference [11] reported a case of rhabdomyolysis that was attributed to oral ritodrine use $(15 \mathrm{mg} /$ day) for tocolysis in a patient with background myotonic dystrophy presenting with preterm contractions, with a surge in serum creatine kinase value to $10,897 \mathrm{mg} / \mathrm{dl}$ and myoglobinuria $(1,800 \mathrm{ng} / \mathrm{dl})$. There were no evident rhabdomyolysis symptoms other than the biochemical derangements in the case they reported.

Reference [12] reported cocaine-induced rhabdomyolysis in a 25-year-old multipara who had abruptio placenta 18 
hours post-cocaine use and needing emergency caesarean section due to ensuing vaginal bleed, foetal acidosis, and bradycardia. Reference [13] reported the case of a 21-yearold a case of baking soda pica (containing $454 \mathrm{~g}$ sodium bicarbonate ingested per day) in a multi-gravida at 31 weeks of pregnancy that caused her severe hypokalemic metabolic alkalosis and rhabdomyolysis (with peak serum creatine kinase value of $32,750 \mathrm{U} / 1$ ), with resultant global limb weakness. She was nursed in an intensive care unit with intravenous fluid therapy and symptoms resolved postdiscontinuation of the baking soda use.

Reference [14] also reported the case of a 35-year-old multigravida who presented at 37 weeks gestational age with generalized weakness and dizziness due to chronic baking soda pica (same daily amount as above) that was complicated by rhabdomyolysis and cardiomyopathy (with echocardiography ejection fraction of $30 \%$ ) and arrhythmias (had runs of ventricular tachycardia with bigeminy and trigeminy as well reported). She was nursed in the intensive care unit and had an induction of labour with assisted forceps delivery.

Studies describing the prevalence or incidence of rhabdomyolysis complicating neuraxial analgesia or anesthesia techniques deployed for different reasons are unavailable, as far as I am aware. Reference [15] published a case series reporting 4 incidences of gluteal compartment syndrome (a possible complication of rhabdomyolysis) in 4 patients (BMI range from $28-38 \mathrm{~kg} / \mathrm{m} 2$ ) after discontinuation of epidural analgesia in the post-operative period for total knee or hip replacement surgeries. The four cases required decompression surgeries.

Reference [16] published a series of 2 cases describing two patients who developed gluteal compartment syndrome after having epidural techniques for total knee replacements and afterwards required fasciotomies. Reference [17] also described a post-traumatic case of bilateral gluteal compartment syndromes requiring fasciotomy. Reference [18] reported a case of an acute compartment syndrome due to superior gluteal artery rupture in a patient following a low energy trauma (fall) and required an emergency fasciotomy. Reference [19] reported a case of gluteal compartment syndrome in a chronic drinker who had prolonged immobility and needed fasciotomy and debridement. Reference [20] reported a case of gluteal compartment syndrome attributed to heroin use.

With the increasing documentations or literatures about epidural analgesia or anaesthesia implicated in gluteal compartment syndrome following lower limb orthopaedic procedures, it remains to be described if the risk of rhabdomyolysis remains a potential threat whenever labour epidural or spinal techniques are deployed to pregnant women in the peri-partum period. Multidisciplinary management involving the obstetric, anaesthesiology, paediatric, internal medicine and chemical pathology units, the intensive care team, together with support of nursing staff, will be needed in the care of a pregnant patient who develops rhabdomyolysis especially in the peri-partum period. The obstetric anesthesiologist should participate from an early point. Continuous monitoring and a readiness to correct blood gases, electrolytes, creatinine, CPK, myoglobin, aldolase, cardiac and urine output monitoring are equally as important.
Adequate hydration to prevent nephrotoxicity caused by myoglobins released from damaged muscles is needed while managing rhabdomyolysis. Cardiotocography for foetal wellbeing assessment is also important.

Advanced diagnostic techniques such as intracompartmental pressure monitoring, and near-infrared spectroscopy (NIRS) can be used to reduce the morbidity that can result from the development of a compartment syndrome complicating rhabdomyolysis. Surgical intervention (fasciotomy with or without debridement) may be required in such severe cases [21].

Serial extended clotting screen evaluation will be needed in the setting of disseminated intravascular coagulopathy (DIC) complicating rhabdomyolysis and blood products may need to be transfused. Renal replacement therapy may be considered in the setting of severe acute kidney injury, severe acidosis and severe hyperkalemia where other measures fail.

A birth plan where rhabdomyolysis complicates the peripartum period would depend on the stage of pregnancy, whether the patient is in labour or not and how severe the rhabdomyolysis is. The decision could be watchful waiting while medical therapy is being deployed or decision to proceed with delivery by spontaneous or induced or via operative means in a centre capable of operative delivery and being able to offer adult and neonatal resuscitations and intensive care service.

Literature on the management of labour complicated by rhabdomyolysis or the development of rhabdomyolysis on labour epidural analgesia administration is scanty, hence, the timeliness of this review article to stimulate further discussion about this important association and possibly serve as a guide for obstetric anesthesiologists to manage future similar cases.

\section{ACKNOWLEDGMENT}

The author wishes to thank Dr. Reddy Shamantha of the Obstetric Anesthesiology Unit at the Montefiore Medical Centre, Bronx, New York, USA, for sharing insights on this topic.

\section{REFERENCES}

[1] P. A. Torres, J. A. Helmstetter, A. M. Kaye and D. K. Alan, "Rhabdomyolysis: pathogenesis, diagnosis, and treatment. Ochsner Journal 15(1): 58-69, 2015.

[2] B. V. Li, D. A. Strong and H. M. Esdale, "Recurrent severe rhabdomyolysis in a pregnancy affected by myotonic dystrophy type 1," Pathology 52, S98, 2020.

[3] J. M. Sauret, G. Marinides and G. K. Wang, "Rhabdomyolysis," American Family Physician 65(5): 907-12, 2002.

[4] R. Momoh, T. Sherica and S. Reddy, "A Case of Rhabdomyolysis in A Pregnant Patient During Labor" https://cdn.technologynetworks.com/ep/pdfs/a-case-ofrhabdomyolysis-in-a-pregnant-patient-during-labor.pdf

[5] N. Petejova and A. Martinek, "Acute kidney injury due to rhabdomyolysis and renal replacement therapy: a critical review," Critical Care 18(3): 224, 2014.

[6] Y. S. Chatzizisis, G. Misirli, A. I. Hatzitolios and G. D. Giannoglou, "The syndrome of rhabdomyolysis: complications and treatment," European Journal of Internal Medicine 19(8): 568-574, 2008.

[7] M. Srisuttayasathien, "A Case of Hypokalemia-Induced Rhabdomyolysis as a result of Distal Renal Tubular Acidosis in a Pregnant Woman: A Case Report and Literature Review," Hindawi Journal Volume 2015. 
[8] G. Carminati, A. Chena, J. M. Orlando, S. Russo, S. Salomón and J. A. Carena, "A case series on the incidences of distal renal tubular acidosis with rhabdomyolysis as the presenting form in 4 pregnant women," https://europepmc.org/article/med/11464655.

[9] Wikipedia contributors. Rhabdomyolysis. Wikipedia, The Free Encyclopedia.

https://en.wikipedia.org/w/index.php?title=Rhabdomyolysis\&oldid=1 041819195.

[10] D. Yürük, A. Yilmaz, G. E. Özgencil and İ. Aşik: “A Case Report: Acute rhabdomyolysis following epidural steroid injection: An unusual complication in a patient with low back pain," Agri 31(3): 150-152, 2019.

[11] K. Nasu, T. Sugano, J. Yoshimatsu and H. Narahara, "Rhabdomyolysis Caused by Tocolysis with Oral Ritodrine Hydrochloride in a Pregnant Patient with Myotonic Dystrophy," Gynecologic and Obstetric Investigation 61(1): 53-55, 2006

[12] E. C. Lampley, S. Williams, S. A. Myers, "Cocaine-associated rhabdomyolysis causing renal failure in pregnancy," Obstetrics \& Gynecology, 87(5): 804-806, 1996.

[13] C. A. Grotegut, V. Dandolu, S. Katari, V. E. Whiteman, O. GeifmanHoltzman and M. Teitelman, "Baking soda pica: a case of hypokalemic metabolic alkalosis and rhabdomyolysis in pregnancy," Obstetrics \& Gynecology 107(2): 484-486, 2006.

[14] K. M. S. Childress and T. Myles, "Baking soda pica associated with rhabdomyolysis and cardiomyopathy in pregnancy," Obstetrics \& Gynecology 122(2): 495-497, 2013.

[15] V. Kumar, K. Saeed, A. Panagopoulos and P. J. Parker, "Gluteal compartment syndrome following joint arthroplasty under epidural anaesthesia: a report of 4 cases," Journal of Orthopaedic Surgery 15(1): 113-117, 2007.

[16] R. J. Pacheco, S. Buckley, N. J. Oxborrow, A. C. Weeber and K. Allerton, "Gluteal compartment syndrome after total knee arthroplasty with epidural postoperative analgesia," The Journal of Bone and Joint Surgery 83(5): 739-740, 2001.

[17] D. Barick and A. Nemade, "Bilateral post-traumatic gluteal compartment syndrome: A case report and review of literature," Journal of Orthopaedics and Allied Sciences, 3(1): 33, 2015.

[18] A.Smith, V. Chitre and H. Deo, "Acute gluteal compartment syndrome: superior gluteal artery rupture following a low energy injury," Case Reports, bcr2012007710, 2012.

[19] H. L. Liu and D. S. Wong, "Gluteal compartment syndrome after prolonged immobilization," Asian Journal of Surgery 32(2): 123-126, 2009.

[20] T. Klockgether, M. Weller, T. Haarmeier, B. Kaskas, G. Maier and J. Dichgans, "Gluteal compartment syndrome due to rhabdomyolysis after heroin abuse," Neurology 48(1): 275-276, 1997.

[21] G. Via, F. Oliva, M. Spoliti and N. Maffulli, "Acute compartment syndrome," Muscles, Ligaments and Tendons Journal 5(1): 18, 2015. 